\title{
Complement inhibitors finally find orphan niches
}

Since the early 1990s, the New England-area companies Alexion Pharmaceuticals of Cheshire, Connecticut, and Avant Immunotherapeutics of Needham, Massachusetts, have been linked by their efforts to develop the first drugs that inhibit the complement cascade, to potentially treat a range of diseases including arthritis and cardiovascular disease, as well as the ancillary care of transplant. The companies have navigated a circuitous route of mixed clinical results, but the endpoints for their respective programs may be in sight.

With the release January 26 of phase 3 data, Alexion appears on track for a filing for marketing approval for its first drug, Soliris (eculizumab), in 2006. If approved, it could become the first drug to treat a rare form of hemolytic anemia called paroxysmal nocturnal hemoglobinuria (PNH). Avant, for its part, now foresees a clear development path for its TP-10 (soluble complement receptor type 1), as a malesonly therapy in cardiac bypass surgery.

Finding the appropriate clinical setting for a complement inhibitor has been challenging. The complement cascade comprises a series of inactive proteins circulating in the blood. Activation of the pathway leads to enzymatic cleavage of single complement proteins and their byproducts, which usually form the next enzymatic complex to cleave the subsequent inactive protein, ultimately leading to the production of anaphylatoxins (C4a, C3a and $\mathrm{C} 5 \mathrm{a}$ ) and opsonin $\mathrm{C} 3 \mathrm{~b}$ (which activates and recruits phagocytic white blood cells) as well as formation of the lytic membrane attack complex (MAC; C5b, C6-C9). As the red blood cells of $\mathrm{PNH}$ patients are depleted of the membrane glycoprotein CD59, a key inhibitor of complement's lytic activity, it seems likely that complement activation plays a role in the erythrolysis that leads to $\mathrm{PNH}$.

"It's difficult to find clinical trials indications that give a clear answer with something as complex as complement inhibition," notes Una Ryan, president and CEO of Avant. "A host of stimuli can initiate this unidirectional cascade," adds Alexion CEO Leonard Bell, including autoimmune diseases, infections, ischemic injury, reperfusion and atherosclerosis.

Initially, Alexion focused on developing Soliris, a humanized monoclonal antibody that binds a portion of complement receptor 5 (C5) and blocks terminal complement activation, and also a single-chain version of the antibody, in acute cardiovascular diseases, autoimmune diseases and $\mathrm{PNH}$, although it did not see commercial value in the last. "For many years, there was a healthy discussion within the company regarding various disease targets," Bell recalls.

The first PNH trial of Soliris, a phase 2 study in 11 patients, showed that it reduced hemolysis and therefore blood transfusions, and increased the size of the PNH red cell clone in patients so that they maintained stable hemoglobin. "It was a good example of compelling data," says Bell. "But from the development perspective, it was only eleven patients in an uncontrolled trial."

By Bell's own admission, Alexion was slow to understand the commercial prospects of ultra-orphan drug development; namely, the commercialization of a drug for a disease that affects less than 10,000 patients in the US. Plus, he adds, "There's no incentive to find out until there's a development to suggest there might be an effective therapeutic," and there were no therapies for PNH. Nonetheless, says Bell, "we decided that we may not know how many patients there are, but the scientific basis for the drug's being effective is substantial.

"I didn't appreciate Genzyme's accomplishments from a commercial point of view as much as I should have," Bell acknowledges. With its Cerezyme (imiglucerase), Fabrazyme (agalsidase beta) and Aldurazyme (laronidase) treatments, all for ultraorphan diseases, Genzyme of Cambridge, Massachusetts, had shown that a biotech company can succeed in very small patient markets with very expensive first-in-class drugs. Summit, New Jersey-based Celgene has made the same point with Thalomid (thalidomide); Novo Nordisk, located in Bagsværd, Denmark, with Novo 7 (recombinant factor VIIa), and Basingstoke, UKbased Shire/Transkaryotic Therapies with Replagal (agalsidase alfa) for Fabry disease,

\section{Box 1 Engaging with the FDA's Special Protocol Assessment scheme}

Alexion tapped into the FDA's Special Protocol Assessment (SPA) process, which offers drug sponsors an opportunity to discuss the design of a pivotal trial in advance with the FDA. SPA is especially useful in diseases for which clinical trial endpoints are not well established. Given the small amount of data it had, going through the more meetingand paperwork-heavy SPA process was helpful to Alexion for several reasons. "We had come into FDA with no US IND [Investigational New Drug Application], data on eleven patients that had three months of therapy, and suggesting it would be appropriate to take it into a conventional pivotal licensing trial," Bell explains.

"And in a clinical setting where there had been no prior drug development, and therefore no established licensable endpoint, what do you pick?" Bell asks. Twelve months later, the agency and the company had settled on the clinical endpoints to use in phase 3. Bell notes that FDA was also "looking to guard us away from an endpoint that could lead to a more complicated regulatory review." Alexion is having similar discussions with the European Medicines Agency for a European approval. Bell points out that the regulators there "frequently take a more liberal view of ultra-orphan disorders." 
which is approved in Europe but not the US. "The attraction to a small company addressing a high-value, low-volume marketplace is extremely high," Bell now contends, based on a small company's smaller infrastructure, which can compete on an equal basis with a larger company.

Whereas Alexion is now focused on obtaining marketing approval and selling Soliris on its own to the very small $\mathrm{PNH}$ patient population, Avant shifted its priorities to vaccine development and identified TP-10 as an outlicensing prospect, making that drug's fate problematic.

One month after Alexion's phase 3 Soliris data came out, Avant announced phase $2 b$ data on TP-10 strongly confirming observations that although it appears effective in treating men who undergo cardiac bypass surgery, it has no beneficial effect in women.

(A previous phase 2 study in cardiac bypass surgery showed strong efficacy in males, but little effect in females.)

\section{"The attraction to a small company addressing a high- value, low-volume marketplace is extremely high," says Alixion CEO Leonard Bell.}

In an interview two weeks before Avant's data disclosure, CEO Ryan addressed the question of gender and the difference in results between men and women. Concerned that Avant's initial phase 2 data showing the drug had no effect in women was only the consequence of small numbers, US Food and Drug Administration (FDA) wouldn't let trials go forward only in men absent an explanation for the difference in results, she explained.

Ryan says that with the new phase 2 data the company can now "aggressively partner TP-10 in cardiac surgery," adding that partners were already lined up. "Some wanted the full label, others felt the regulatory pathway was much clearer if the trial confirmed the previous result [in women]," she says. Indeed, precedent already exists for such a subgroup-specific approval by the FDA: witness the recent example of Nitromed, which last year won FDA approval to market BiDil (isosorbide dinitrate/hydralazine hydrochloride) specifically to treat heart attacks in African-Americans.

Mark Ratner, Cambridge, Massachusetts 